\title{
Stability Analysis of a Delayed HIV/AIDS Epidemic Model with Treatment and Vertical Transmission
}

\author{
Zohragul Osman, Xamxinur Abdurahman* \\ College of Mathematics and System Sciences, Xinjiang University, Urumqi, China \\ Email: xamxinur@sina.com
}

Received 7 April 2015; accepted 22 September 2015; published 25 September 2015

Copyright (C) 2015 by authors and Scientific Research Publishing Inc.

This work is licensed under the Creative Commons Attribution International License (CC BY).

http://creativecommons.org/licenses/by/4.0/

(c) (i) Open Access

\begin{abstract}
A delayed HIV/AIDS epidemic model with treatment and vertical transmission is investigated. The model allows some infected individuals to move from the symptomatic phase to the asymptomatic phase; next generation of infected individuals may be infected and it will take them some time to get maturity and infect others. Mathematical analysis shows that the global dynamics of the spread of the HIV/AIDS are completely determined by the basic reproduction number $R_{0}$ for our model. If $R_{0}<1$ then disease free equilibrium is globally asymptotically stable, whereas the unique infected equilibrium is globally asymptotically stable if $R_{0}>1$.
\end{abstract}

\section{Keywords}

HIV/AIDS Epidemic Model, Vertical Transmission, Basic Reproduction Number, Time Delay

\section{Introduction}

Mathematical models play an important role in the study of the transmission dynamics of HIV/AIDS, and in some sense, delay models give better compatibility with reality, as they capture the dynamics from the time of infection to the infectiousness. Some HIV/AIDS models are introduced in [1]-[5]. In recent years, a few studies of vertical transmission have been conducted to describe the effects of various epidemiological and demographical factors [6]-[8], and some models considered vertical transmission with time delay [9] [10]. Some specific $H I V$ models with imperfect vaccine were introduced in [11]-[13].

In [1], L. Cai and X. Li studied local and global stability of the equilibria of a SIJA model with treatment:

${ }^{*}$ Corresponding author. 


$$
\left\{\begin{array}{l}
\frac{\mathrm{d} S}{\mathrm{~d} t}=\mu K-c \beta(I+b J) S-\mu S, \\
\frac{\mathrm{d} I}{\mathrm{~d} t}=c \beta(I+b J) S-\left(\mu+k_{1}\right) I+\alpha J, \\
\frac{\mathrm{d} J}{\mathrm{~d} t}=k_{1} I-\left(\mu+k_{2}+\alpha\right) J \\
\frac{\mathrm{d} A}{\mathrm{~d} t}=k_{2} J-(\mu+d) A .
\end{array}\right.
$$

In model (1), it is assumed that some individuals with the symptomatic phases $J$ can be transformed into asymptomatic individuals $I$ after treatment and they get the result that when $R_{0}<1$ the disease free equilibrium is globally asymptotically stable and if $R_{0}>1$ the endemic equilibrium is globally asymptotically stable.

In [9], Ram Naresh et al. considered the following SIA model with vertical transmission:

$$
\left\{\begin{array}{l}
\frac{\mathrm{d} S}{\mathrm{~d} t}=Q_{0}-\frac{\beta S I}{N}-d S, \\
\frac{\mathrm{d} I}{\mathrm{~d} t}=\frac{\beta S I}{N}-(d+\delta) I+\gamma \epsilon I(t-\tau) \mathrm{e}^{-d \tau}, \\
\frac{\mathrm{d} A}{\mathrm{~d} t}=\delta I+\gamma(1-\epsilon) I(t-\tau) \mathrm{e}^{-d \tau}-(\alpha+d) A .
\end{array}\right.
$$

Here, the authors assume that a fraction of newborns, who sustain treatment, join the infective class while others, who do not sustain treatment, join the AIDS class after getting sexual maturity. The infectives through vertical transmission at any time $t$ are given by $\gamma \epsilon I(t-\tau) \mathrm{e}^{-d \tau}$. The authors proved the local and global stability of disease free equilibrium and endemic equilibrium under some conditions. Inspired by these works, we consider an HIVIAIDS model with vertical transmission and with time delay.

The organization of the paper is as follows. In the next section we present the model with delay. Section 3 presents the basic properties of the model. In Section 4, we analyze local and global stability of equilibrium points. In the last section, we present a brief conclusion.

\section{Mathematical Model}

We propose an HIV/AIDS model which incorporates time delay during which a newly born infected child attains sexual maturity and becomes infectious. In this model, the sexually mature population is divided into four subclasses: the susceptibles $(S)$, the asymptomatic infectives $(I)$, the symptomatic infectives $(J)$ and full-blown AIDS group (A). The number of total population is denoted by $N(t)$, for any time $t$. We assume that the susceptibles become HIV infected via sexual contacts with infectives. It is also assumed that all newborns are infected at birth $(\tau=0, p=1)$. It is reasonable to assume that full-blown AIDS patients are sexually inactive and symptomatic stage patients feel uncomfortable (some may know they are AIDS) and the possibility of producing children is small, so can be taken negligible. We also assume that a fraction of infected newborns, who sustain treatment, joins the asymptomatic infective class while others, who do not sustain treatment, joins AIDS class after getting sexual maturity. The infectives through vertical transmission at any time $t$ is given by $\gamma p I(t-\tau)$, because those who are infected at time $(t-\tau)$ becomes infectoius (asymptomatic stage infectious) at time $t$, if they do not develop to AIDS patient by that time. The fraction of infectives which became AIDS patient during the period of getting sexual maturity, if they survive to the maturity, joins to the AIDS class. However, for the model to be biologically reasonable, it may be more realistic to assume that not all those infected will survive after $\tau$ time units, and this claim support the introduction of the survival term $\mathrm{e}^{-\mu \tau}$. Thus, in our model the term $\gamma p I(t-\tau) \mathrm{e}^{-\mu \tau}$ also represents the introduction of infectives through vertical transmission. If the birth rate of newborns $\gamma$ equals to zero, then our model will back to the model (1).

With the above considerations and assumptions, the spread of the disease is assumed to be governed by the following model: 


$$
\left\{\begin{array}{l}
\frac{\mathrm{d} S}{\mathrm{~d} t}=\mu K-c \beta[I(t)+b J(t)] S(t)-\mu S(t), \\
\frac{\mathrm{d} I}{\mathrm{~d} t}=c \beta[I(t)+b J(t)] S(t)-\left(\mu+k_{1}\right) I(t)+\alpha J(t)+\gamma p I(t-\tau) \mathrm{e}^{-\mu \tau}, \\
\frac{\mathrm{d} J}{\mathrm{~d} t}=k_{1} I(t)-\left(\mu+k_{2}+\alpha\right) J(t), \\
\frac{\mathrm{d} A}{\mathrm{~d} t}=k_{2} J(t)-(\mu+d) A(t)+(1-p) \gamma I(t-\tau) \mathrm{e}^{-\mu \tau}
\end{array}\right.
$$

where $\mu K$ is the recruitment rate of the population, $\mu$ is the death rate. $c$ is the average number of contacts of an individual per unit of time. $\beta$ and $b \beta$ are the probability of disease transmission per contact by an infective in the first stage and in the second stage, respectively. $k_{1}$ and $k_{2}$ are transfer rate from the asymptomatic phase $I$ to the symptomatic phase $J$ and from the symptomatic phase to the AIDS cases, respectively. $\alpha$ is transformation rate from the symptomatic phase $J$ to asymptomatic phase $I . d$ is the disease-related death rate of the AIDS cases. $\gamma$ is the birth rate of infected newborns, $p$ is the fraction of infected newborns joining the asymptomatic infective class after getting sexual maturity and remaining part $(1-p)$ of the infected newborns joins the AIDS class after getting sexual maturity $(0 \leq p \leq 1)$. It is also assumed that all the parameters of the model are non-negative. Based on it's biological meaning, we always assume that $k_{2} \geq k_{1}$.

\section{Basic Properties}

For model (2), let the initial condition be $S(0)=S_{0}>0, I(\theta)=I_{\theta} \geq 0, J(0)=J_{0} \geq 0, A(0)=A_{0} \geq 0$ for all $\theta \in[-\tau, 0]$, with $I_{0}(0)>0$. Then, it is clear that the solution $(S(t), I(t), J(t), A(t))$ of the model (3) remain positive for all time $t>0$.

Let $N(t)=S(t)+I(t)+J(t)+A(t)$, then

$$
N(t)^{\prime}=\mu K-\mu N(t)-d A(t)+\gamma I(t-\tau) \mathrm{e}^{-\mu \tau} \leq \mu K-\mu N(t)+\gamma N(t)
$$

which gives,

$$
\lim _{t \rightarrow \infty} \sup N(t)=\frac{\mu K}{\mu-\gamma}
$$

Define

$$
\Omega=\left\{(S, I, J, A): S(t)+I(t)+J(t)+A(t) \leq \frac{\mu K}{\mu-\gamma}, S(t)>0, I(t) \geq 0, J(t) \geq 0, A(t) \geq 0\right\} .
$$

This implies that if $\mu>\gamma$ all solutions of model (3) starting in $R_{+}^{3}$ are bounded and eventually enter the attracting set $\Omega$.

It is reasonable to assume that the general death rate $\mu$ is greater than the birth rate of infected newborns $\gamma$, that is $\mu>\gamma$. In some models, death rate equal to birth rate. However, in this model, $\gamma$ is smaller than birth rate. Below we assume $\mu>\gamma$.

Since the variable A of model (3) does not appear in the first three equation, in the subsequent analysis, we only consider the submodel:

$$
\left\{\begin{array}{l}
\frac{\mathrm{d} S}{\mathrm{~d} t}=\mu K-c \beta[I(t)+b J(t)] S(t)-\mu S(t), \\
\frac{\mathrm{d} I}{\mathrm{~d} t}=c \beta[I(t)+b J(t)] S(t)-\left(\mu+k_{1}\right) I(t)+\alpha J(t)+\gamma p I(t-\tau) \mathrm{e}^{-\mu \tau} \\
\frac{\mathrm{d} J}{\mathrm{~d} t}=k_{1} I(t)-\left(\mu+k_{2}+\alpha\right) J(t) .
\end{array}\right.
$$

Model (4) always has a disease-free equilibrium $E_{0}=(K, 0,0)$. Further we define the basic reproduction 
number $R_{0}$ as follows.

$$
R_{0}=\frac{c \beta K\left(\mu+k_{2}+\alpha+b k_{1}\right)}{\left(\mu+k_{1}\right)\left(\mu+k_{2}\right)+\alpha \mu-\left(\mu+k_{2}+\alpha\right) \gamma p \mathrm{e}^{-\mu \tau}} .
$$

By straightforward computation, when $R_{0}>1$ model (4) has the unique positive equilibrium $E^{*}\left(S^{*}, I^{*}, J^{*}\right)$, where

$$
\begin{aligned}
& S^{*}=\frac{\left(\mu+k_{1}\right)\left(\mu+k_{2}\right)+\alpha \mu-\left(\mu+k_{2}+\alpha\right) \gamma p \mathrm{e}^{-\mu \tau}}{c \beta\left(\mu+k_{2}+\alpha+b k_{1}\right)}=\frac{K}{R_{0}}, \\
& I^{*}=\frac{\left(\mu+k_{2}+\alpha\right) \mu K\left(1-\frac{1}{R_{0}}\right)}{\left(\mu+k_{1}\right)\left(\mu+k_{2}\right)+\alpha \mu-\left(\mu+k_{2}+\alpha\right) \gamma p \mathrm{e}^{-\mu \tau}}, \\
& J^{*}=\frac{k_{1} I^{*}}{\mu+k_{2}+\alpha} .
\end{aligned}
$$

\section{Stability Analysis}

First we will study the local and global stability of disease free equilibrium $E_{0}$.

The variational matrix of model (4) is given by

$$
J=\left(\begin{array}{ccc}
c \beta(I+b J)-\mu & -c \beta S & -b c \beta S \\
c \beta(I+b J) & c \beta S-\left(\mu+k_{1}\right)+\gamma p \mathrm{e}^{-(\mu+\lambda) \tau} & b c \beta S+\alpha \\
0 & k_{1} & -\left(\mu+k_{2}+\alpha\right)
\end{array}\right) .
$$

Theorem 4.1. If $R_{0}<1$, the disease free equilibrium $E_{0}$ is locally asymptotically stable.

Proof. The Jacobian matrix corresponding to model (4) about $E_{0}$ as follows,

$$
J\left(E_{0}\right)=\left(\begin{array}{ccc}
-\mu & -c \beta K & -b c \beta K \\
0 & c \beta K-w+\gamma p \mathrm{e}^{-(\mu+\lambda) \tau} & b c \beta K+\alpha \\
0 & k_{1} & -(v+\alpha)
\end{array}\right),
$$

where $w=\mu+k_{1}, \quad v=\mu+k_{2}$.

The characteristic equation of this matrix is given by $\operatorname{det}\left(\lambda I-J\left(E_{0}\right)\right)=0$, where $I$ is the unit matrix.

$$
(\lambda+\mu)\left[\lambda^{2}+a_{1} \lambda+a_{0}+\left(b_{1} \lambda+b_{0}\right) \mathrm{e}^{-\lambda \tau}\right]=0,
$$

where

$$
\begin{aligned}
& a_{1}=w+v+\alpha-c \beta K, \\
& a_{0}=(v+\alpha)(w-c \beta K)-(b c \beta K+\alpha) k_{1}, \\
& b_{1}=-\gamma p \mathrm{e}^{-\mu \tau}, \\
& b_{0}=-(v+\alpha) \gamma p \mathrm{e}^{-\mu \tau} .
\end{aligned}
$$

Clearly, one root of this equation is $\lambda=-\mu$. So we consider the following equation.

$$
\lambda^{2}+a_{1} \lambda+a_{0}+\left(b_{1} \lambda+b_{0}\right) \mathrm{e}^{-\lambda \tau}=0 .
$$

If $\tau=0$, the equation becomes

$$
\lambda^{2}+\left(a_{1}+b_{1}\right) \lambda+a_{0}+b_{0}=0
$$


Since

$$
\begin{aligned}
& a_{0}+b_{0}=w v+\alpha \mu-(v+\alpha) \gamma p \mathrm{e}^{-\mu \tau}-c \beta K\left(v+\alpha+b k_{1}\right), \\
& a_{1}+b_{1}=w+v+\alpha-c \beta K-\gamma p \mathrm{e}^{-\mu \tau},
\end{aligned}
$$

when $R_{0}<1, a_{1}+b_{1}>0, a_{0}+b_{0}>0$, notice that $\lambda_{1}+\lambda_{2}=-\left(a_{1}+b_{1}\right)<0, \lambda_{1} \lambda_{2}=a_{0}+b_{0}>0$. Hence the roots of this equation have negative real part by the Hurwitz criterion.

If $\tau>0$, we assume that $\lambda=i \omega(\omega>0)$ is the root of characteristic Equation (6), then $\omega$ satisfies

$$
-\omega^{2}+a_{1} i \omega+a_{0}=\left(-b_{1} i \omega-b_{0}\right)(\cos \omega \tau-i \sin \omega \tau) .
$$

Separating the real and imaginary parts, we have

$$
\begin{aligned}
& -\omega^{2}+a_{0}=-b_{1} \omega \sin \omega \tau-b_{0} \cos \omega \tau, \\
& a_{1} \omega=-b_{1} \omega \cos \omega \tau+b_{0} \sin \omega \tau .
\end{aligned}
$$

Eliminating $\tau$ by squaring and adding above the two equation, we get that

$$
\omega^{4}+\left(a_{1}^{2}-2 a_{0}-b_{1}^{2}\right) \omega^{2}+a_{0}^{2}-b_{0}^{2}=0 .
$$

Let $p=\omega^{2}$, then this equation becomes

$$
p^{2}+\left(a_{1}^{2}-2 a_{0}-b_{1}^{2}\right) p+a_{0}^{2}-b_{0}^{2}=0 .
$$

Through simple computation, we can found that all the coefficients of this equation is positive, so Equation (7) have no solution, it implies that Equation (6) have not the root like $\lambda=i \omega$. Hence all roots of (6) have negative real part.

We are now in a position to investigate the global stability of the disease-free equilibrium $E_{0}$.

Theorem 4.2. If $R_{0}<1$, then the infection free equilibrium $E_{0}$ is globally asymptotically stable.

Proof. Consider the following Lyapunov functional.

$$
L=\left(\mu+k_{2}+\alpha+b k_{1}\right) I(t)+\left[b\left(\mu+k_{1}-\gamma p \mathrm{e}^{-\mu \tau}\right)+\alpha\right] J(t)+\left(\mu+k_{2}+\alpha+b k_{1}\right) \gamma p \mathrm{e}^{-\mu \tau} \int_{t-\tau}^{t} I(\theta) \mathrm{d} \theta .
$$

Calculating the derivative of $L$ along with the solution of model (4), we have

$$
\begin{aligned}
L^{\prime}= & \left(\mu+k_{2}+\alpha+b k_{1}\right)\left\{c \beta[I(t)+b J(t)] S(t)-\left(\mu+k_{1}\right) I(t)+\alpha J(t)+\gamma p I \mathrm{e}^{-\mu \tau}\right\} \\
& +\left[b\left(\mu+k_{1}-\gamma p \mathrm{e}^{-\mu \tau}\right)+\alpha\right]\left(k_{1} I(t)-\left(\mu+k_{2}+\alpha\right) J(t)\right) \\
= & {\left[\left(\mu+k_{2}+\alpha+b k_{1}\right) c \beta S(t)-\left(\left(\mu+k_{1}\right)\left(\mu+k_{2}\right)+\alpha \mu-\left(\mu+k_{2}+\alpha\right) \gamma p \mathrm{e}^{-\mu \tau}\right)\right][I(t)+b J(t)] } \\
= & {\left[\left(\mu+k_{1}\right)\left(\mu+k_{2}\right)+\alpha \mu-\left(\mu+k_{2}+\alpha\right) \gamma p \mathrm{e}^{-\mu \tau}\right]\left(R_{0}-1\right)[I(t)+b J(t)] . }
\end{aligned}
$$

This implies that $\frac{\mathrm{d} L}{\mathrm{~d} t} \leq 0$ when $R_{0}<1$, the equality $L^{\prime}=0$ holds if and only if $I=0, J=0$, the maximal invariant set of $\left\{(S, I, R): L^{\prime}=0\right\}$ is the singleton $\left\{E_{0}\right\}$. Hence $E_{0}$ is globally asymptotically stable by the LaSalle invariance principle [14].

Now, when $R_{0}>1$ we will study the local and global stability of $E^{*}$.

Theorem 4.3. If $R_{0}>1$, the infected equilibrium $E^{*}$ is locally asymptotically stable.

Proof. For this purpose, we obtain the Jacobian matrix corresponding to model (4) about $E^{*}$ as follows,

$$
J\left(E^{*}\right)=\left(\begin{array}{ccc}
-q-\mu & -c \beta S^{*} & -b c \beta S^{*} \\
q & c \beta S^{*}-w+\gamma p \mathrm{e}^{-(\mu+\lambda) \tau} & b c \beta S^{*}+\alpha \\
0 & k_{1} & -(v+\alpha)
\end{array}\right),
$$


where $w=\mu+k_{1}, \quad v=\mu+k_{2}, \quad q=c \beta\left(I^{*}+b J^{*}\right)$.

The characteristic equation of this matrix is

$$
\lambda^{3}+m_{2} \lambda^{2}+m_{1} \lambda+m_{0}+\left(n_{2} \lambda^{2}+n_{1} \lambda+n_{0}\right) \mathrm{e}^{-\lambda \tau}=0,
$$

where

$$
\begin{aligned}
& m_{2}=w-c \beta S^{*}+v+\alpha+\mu+q, \\
& m_{1}=(v+\alpha)(\mu+q)+\left(w-c \beta S^{*}\right)(v+\alpha+\mu+q)-k_{1}\left(\alpha+b c \beta S^{*}\right)+c \beta q S^{*}, \\
& m_{0}=(v+\alpha)(\mu+q)\left(w-c \beta S^{*}\right)-k_{1}(\mu+q)\left(\alpha+b c \beta S^{*}\right)+(v+\alpha) c \beta q S^{*}+k_{1} b c \beta q S^{*}, \\
& n_{2}=-\gamma p \mathrm{e}^{-\mu \tau} \\
& n_{1}=-\gamma p \mathrm{e}^{-\mu \tau}(v+\alpha+\mu+q), \\
& n_{0}=-\gamma p \mathrm{e}^{-\mu \tau}(v+\alpha)(\mu+q) .
\end{aligned}
$$

Notice that

$$
(v+\alpha)\left(w-c \beta S^{*}\right)-k_{1}\left(\alpha+b c \beta S^{*}\right)=(v+\alpha) \gamma p \mathrm{e}^{-\mu \tau} .
$$

Hence

$$
\begin{aligned}
& m_{1}=(v+\alpha)(\mu+q)+(\mu+q)\left(w-c \beta S^{*}\right)+(v+\alpha) \gamma p \mathrm{e}^{-\mu \tau}+c \beta q S^{*}>0, \\
& m_{2}=(v+\alpha)(\mu+q) \gamma p \mathrm{e}^{-\mu \tau}+(v+\alpha) c \beta q S^{*}+k_{1} b c \beta q S^{*}
\end{aligned}
$$

when $\tau=0$, the characteristic Equation (8) yields

$$
\lambda^{3}+l_{2} \lambda^{2}+l_{1} \lambda+l_{0}=0
$$

where

$$
\begin{aligned}
& l_{2}=w-c \beta S^{*}+v+\alpha+\mu+q-\gamma p>0 . \\
& l_{1}=(v+\alpha)(\mu+q)+(\mu+q)\left(w-c \beta S^{*}-\gamma p\right)+(v+\alpha) \gamma p \mathrm{e}^{-\mu \tau}+c \beta q S^{*}>0 . \\
& l_{0}=(v+\alpha) c \beta q S^{*}+k_{1} b c \beta q S^{*}>0 .
\end{aligned}
$$

Obviously, $l_{0}>0, l_{1}>0, l_{2}>0$ and $l_{1} l_{2}-l_{0}>0$. This implies that when $R_{0}>1$ and $\tau=0, E^{*}$ is locally asymptotically stable by the Hurwitz criterion.

Now we study the stability behavior of $E^{*}$ in the case $\tau>0$.

We assume that $\lambda=i \eta(\eta>0)$ is the root of characteristic equation, then $\eta$ satisfies

$$
-i \eta^{3}-m_{2} \eta^{2}+m_{1} i \eta+m_{0}=\left(n_{2} \eta^{2}-n_{1} i \eta-n_{0}\right)(\cos \eta \tau-i \sin \eta \tau) .
$$

Separating the real and imaginary parts, we have

$$
\begin{aligned}
& -m_{2} \eta^{2}+m_{0}=\left(n_{2} \eta^{2}-n_{0}\right) \cos \eta \tau-n_{1} \eta \sin \eta \tau, \\
& -\eta^{3}+m_{1} \eta=-\left(n_{2} \eta^{2}-n_{0}\right) \sin \eta \tau-n_{1} \eta \cos \eta \tau .
\end{aligned}
$$

Eliminating $\tau$ by squaring and adding (9) and (10), we get the equation determining for $\eta$ as,

$$
\eta^{6}+d_{2} \eta^{4}+d_{1} \eta^{2}+d_{0}=0
$$

where

$$
d_{2}=m_{2}^{2}-2 m_{1}-n_{2}^{2}, d_{1}=-2 m_{0} m_{2}+m_{1}^{2}+2 n_{0} n_{2}-n_{1}^{2}, d_{0}=m_{0}^{2}-n_{0}^{2}
$$


Substituting $\eta^{2}=u$ in above Equation, we have

$$
u^{3}+d_{2} u^{2}+d_{1} u+d_{0}=0
$$

when $k_{2} \geq k_{1}$, through simple computation we can see that $d_{0}>0, d_{1}>0, d_{2}>0$, in this circumstance (11) has not positive root. So all roots of (8) has negative real part.

Next, we consider the global stability of $E^{*}$ when $R_{0}>1$.

Theorem 4.4. If $R_{0}>1$, then the infected equilibrium $E^{*}$ is globally asymptotically stable.

Proof. Firstly, we define a function, $g(\xi)=\xi-1-\ln \xi, \xi \in R^{+}$. Take the Lyapunov functional as follows.

$$
V=S^{*} g\left(\frac{S}{S^{*}}\right)+I^{*} g\left(\frac{I}{I^{*}}\right)+M J^{*} g\left(\frac{J}{J^{*}}\right)+I^{*} \gamma p \mathrm{e}^{-\mu \tau} \int_{t-\tau}^{t} g\left(\frac{I(s)}{I^{*}}\right) \mathrm{d} s .
$$

Next calculating the derivative of $V$ along with the solution of model (4), we have

$$
\begin{aligned}
V^{\prime}= & \left(1-\frac{S^{*}}{S}\right)\left[\mu S^{*}+c \beta\left(I^{*}+b J^{*}\right) S^{*}-\mu S-c \beta(I+b J) S\right] \\
& +\left(1-\frac{I^{*}}{I}\right)\left[c \beta(I+b J) S-\frac{c \beta\left(I^{*}+b J^{*}\right) S^{*}+\alpha J^{*}}{I^{*}} I+\alpha J\right] \\
& +M\left(1-\frac{J^{*}}{J}\right)\left[k_{1} I-\frac{k_{1} I^{*}}{J^{*}} J\right]-\gamma p I^{*} \mathrm{e}^{-\mu \tau} g\left(\frac{I(t-\tau)}{I(t)}\right) .
\end{aligned}
$$

Since $g(\xi) \geq 0$, we have

$$
\begin{aligned}
V^{\prime} \leq & \left(1-\frac{S^{*}}{S}\right)\left[\mu S^{*}+c \beta\left(I^{*}+b J^{*}\right) S^{*}-\mu S-c \beta(I+b J) S\right] \\
& +\left(1-\frac{I^{*}}{I}\right)\left[c \beta(I+b J) S-\frac{c \beta\left(I^{*}+b J^{*}\right) S^{*}+\alpha J^{*}}{I^{*}} I+\alpha J\right] \\
& +M\left(1-\frac{J^{*}}{J}\right)\left[k_{1} I-\frac{k_{1} I^{*}}{J^{*}} J\right] .
\end{aligned}
$$

Next,we consider the following variables substitutions by letting,

$$
\frac{S}{S^{*}}=x, \frac{I}{I^{*}}=y, \frac{J}{J^{*}}=z .
$$

Then,

$$
\begin{aligned}
V^{\prime} \leq & -\mu S^{*} \frac{(1-x)^{2}}{x}+2 c \beta I^{*} S^{*}+2 c \beta b J^{*} S^{*}+\alpha J^{*}+M k_{1} I^{*}+x y\left(-c \beta I^{*} S^{*}+c \beta I^{*} S^{*}\right) \\
& +\frac{1}{x}\left(-c \beta I^{*} S^{*}-c \beta b J^{*} S^{*}\right)+y\left[c \beta I^{*} S^{*}-c \beta I^{*} S^{*}-c \beta b J^{*} S^{*}-\alpha J^{*}+M k_{1} I^{*}\right] \\
& +x z\left(-c \beta b J^{*} S^{*}+c \beta b J^{*} S^{*}\right)+z\left[c \beta b J^{*} S^{*}+\alpha J^{*}-M k_{1} I^{*}\right]+x\left(-c \beta I^{*} S^{*}\right) \\
& +\frac{x z}{y}\left(-c \beta b J^{*} S^{*}\right)+\frac{z}{y}\left[-\alpha J^{*}\right]+\frac{y}{z}\left(-M k_{1} I^{*}\right) .
\end{aligned}
$$

Further let

$$
M=\frac{c \beta b J^{*} S^{*}+\alpha J^{*}}{k_{1} I^{*}}
$$


Then, through a straight computation, we have

$$
V^{\prime} \leq-\mu S^{*} \frac{(1-x)^{2}}{x}+c \beta I^{*} S^{*}\left(2-\frac{1}{x}-x\right)+\alpha J^{*}\left(2-\frac{z}{y}-\frac{y}{z}\right)+c \beta b J^{*} S^{*}\left(3-\frac{1}{x}-\frac{x z}{y}-\frac{y}{z}\right) .
$$

Since the arithmetic mean is greater than or equal to the geometric mean and function $g$ is a positive function, we have

$$
\frac{1}{x}+x \geq 2, \quad \frac{z}{y}+\frac{y}{z} \geq 2, \frac{1}{x}+\frac{x z}{y}+\frac{y}{z} \geq 3
$$

Thus, $V^{\prime} \leq 0$ in $\Omega$. The equality $V^{\prime}=0$ holds if and only if $x=y=z=1$. That is, $S=S^{*}, I=I^{*}, J=J^{*}$. The maximal invariant set of model (4) on the set $\left\{(x, y, z): V^{\prime}=0\right\}$ is the singleton $\left\{E^{*}\right\}$. Thus, the endemic equilibrium $E^{*}$ is globally asymptotically stable if $R_{0}>1$ by LaSalle Invariance Principle [14].

\section{Conclusion}

In this paper, we have considered an HIVIAIDS model with treatment, vertical transmission and time delay. Under the assumption that asymptomatic infectives $(J)$ have the symptoms of AIDS, AIDS patients $(A)$ are isolated; hence their probability of producing children is small; and it is neglected. From the local stability of disease free equilibrium, we calculated the basic reproduction number $R_{0}$. Further we get the results that when $R_{0}<1$ the disease free equilibrium is globally asymptotic stable, and when $R_{0}>1$ the endemic equilibrium is globally asymptotic stable.

\section{Acknowledgements}

This work was supported by the National Natural Science Foundation of China (Grants nos. 11261056, 11261058 and 11271312).

\section{References}

[1] Cai, L.M. and Li, X.Z. (2009) Stability Analysis of an HIV/AIDS Epidemic Model with Treatment. Journal of Computational and Applied Mathematics, 229, 313-323. http://dx.doi.org/10.1016/j.cam.2008.10.067

[2] Cai, L.M. and Guo, S.L. (2014) Analysis of an Extended HIV/AIDS Epidemic Model with Treatment. Applied Mathematics and Computation, 236, 621-627. http://dx.doi.org/10.1016/j.amc.2014.02.078

[3] Huo, H.-F. and Feng, L.-X. (2013) Global Stability for an HIV/AIDS Epidemic Model with Different Latent Stages and Treatment. Applied Mathematical Modeling, 37, 1480-1489. http://dx.doi.org/10.1016/j.apm.2012.04.013

[4] Elaiw, A.M. (2010) Global Properties of a Class of HIV Models. Nonlinear Analysis: Real World Applications, 11, 2253-2263. http://dx.doi.org/10.1016/j.nonrwa.2009.07.001

[5] Xiao, D.M. and Ruan, S.G. (2007) Global Analysis of an Epidemic Model with Non-Monotone Incidence Rate. Mathematical Biosciences, 208, 419-429. http://dx.doi.org/10.1016/j.mbs.2006.09.025

[6] Naresh, R., Tripathi, A. and Omar, S. (2006) Modeling the Spread of AIDS Epidemic with Vertical Transmission. Applied Mathematics and Computation, 178, 262-272. http://dx.doi.org/10.1016/j.amc.2005.11.041

[7] d'Onofrio, A. (2005) On Pulse Vaccination Strategy in the SIR Epidemic Model with Vertical Transmission. Applied Mathematics Letters, 18, 729-732. http://dx.doi.org/10.1016/j.aml.2004.05.012

[8] Li, M.Y. and Smith, H.L. (2001) Global Dynamics of an SEIR Epidemic Model with Vertical Transmission. SIAM Journal of Applied Mathematics, 62, 58-69. http://dx.doi.org/10.1137/S0036139999359860

[9] Naresh, R. and Sharma, D. (2011) An HIV/AIDS Model with Vertical Transmission and Time Delay. World Journal of Modeling and Simulation, 7, 230-240.

[10] Liu, J.L. and Zhang, T.L. (2012) Global Stability for Delay SIR Epidemic Model with Vertical Transmission. Open Journal of Applied Sciences, 2, 1-4. http://dx.doi.org/10.4236/ojapps.2012.24b001

[11] Gumel, A.B., McCluskey, C.C. and van den Driessche, P. (2006) Mathematical Study of a Staged-Progression HIV Model with Imperfect Vaccine. Bulletin of Mathematical Biology, 68, 2105-2128. http://dx.doi.org/10.1007/s11538-006-9095-7

[12] Cai, L.M., Fang, B. and Li, X.Z. (2014) A Note of a Staged Progression HIV Model with Imperfect Vaccine. Applied 
Mathematics and Computation, 234, 412-416. http://dx.doi.org/10.1016/j.amc.2014.01.179

[13] McCluskey, C.C. and vanden Driessche, P. (2004) Global Analysis of Two Tuberculosis Models. Journal of Dynamics and Differential Equations, 16, 139-166. http://dx.doi.org/10.1023/B:JODY.0000041283.66784.3e

[14] LaSalle, J.P. (1976) The Stability of Dynamical Systems. In: Regional Conference Series in Applied Mathematics. SIAM, Philadelphia. 